\title{
Research on Psychological Mechanism of Language Transfer in Consecutive Interpreting
}

\author{
Li Wang \\ Foreign Languages College, Qingdao University of Science \& Technology, 266061, China
}

Keywords: Consecutive interpreting, Language transfer, Psychological mechanism

\begin{abstract}
Consecutive interpreting, as a whole, is interpreter behavior of complete and efficient transfer of language after the speaker stop producing source language. In the practical work, English speaker is not only influenced by foreign culture, language skills, language knowledge, but also to some extent by the knowledge representation, perception, memory, attention and other aspects of psychological factors. In the study of the psychological mechanism of language transfer, the paper puts forward the method of asymmetric finite parallel processing. The degree of language similarity and parallel processing is closely related to the language proficiency. Improving the level of language fluency can improve the quality of interpreting, and can effectively control the output of target language and understand the source language.
\end{abstract}

\section{Introduction}

Interpreting can be divided into three stages, understanding of the source language, language transfer and the output of target language, of which the most concerned is language transfer. Only by understanding the nature of language transfer and the relationship between the output of target language the and understanding of source language, can we better carry out the language transfer. On this basis, a certain model is established, which can fully explain the language transfer and predict the influence of interpreting training and the dual language level on environment language.

\section{Language Transfer, Parallel and serial, and Deverbalization}

The study of interpretation an be divided into two categories, the one emphasizing on the scientific nature, mainly by using empirical research methods, and the other focusing on humanities, mainly by adopting the methods of reflection, demonstration, qualitative and interpretation. The above two methods can distinguish between the two concepts of language transfer. Based on the study of humanity, there two ways of transferring the source language, that is, code transfer and deverbalization, which is the main part. In other words, the interpreter can well remember all subtle differences between different sentences, and express them completely and effectively. It is a basic ability to remember the content out of the original form when the source language is about to disappear. It is a way of deep processing and understanding, the same as the normal way. The study emphasizing natural science is to analyze the online transfer of interpreting language, which can be divided into parallel and serial. Different processing methods will form different problems. deverbalization is actually to express the source language form, paying attention to the producing stage. In practical application, the interpreter needs to combine the extra-linguistic knowledge and obtain a certain source language information, and re-express the source language. So it has a relatively small impact. In code transfer, the structure and words of the source language need to be replaced directly, and it has great influence on expressing target language. The main research is the performance of interpretation, and the way to achieve high quality is deverbalization ${ }^{[1]}$.

\section{Asymmetric Finite Parallel Processing Model}

Parallel and serial main focus on two states language transfer: activation and inhibition. The parallel approach thinks that understanding the processing of source language can properly activate the target language, match with the target language in terms of syntactic or lexical level, and transfer 
language at the beginning of understanding source language. So, understanding language, language transfer and output is a process of mutual influence and interaction. The serial is mainly about output and understanding. The processing phase only involves one language, transferring the two languages in a non-verbal way, which is based on the sequence of non-interference, serial processing, conversion and language outputting . The process of interpreting language transfer is in accordance with the asymmetric finite parallel processing model. The parallel means that two interpreted languages are highly activated. At least, a professional interpreter or a fluent bilingual is needed. So, in understanding source language, bilingual parallel is normal; that is to say, in the practice of interpreting, the speed of language input, in a certain extent, leads the lexical chunks to be processed incompletely, and the target language and the source language to be matched incompletely; being asymmetric means that many factors, such as working memory and interpreting training language level, influence the degree of processing. This model can reflect the language transfer mechanism, and has a certain universality. It can be analyzed from the following aspects:

First, in the process of interpretation, the relationship between the two languages can affect the degree of language transfer to a certain extent. A large amount of information says that, in English-Spanish translation, the homologous words can promote the speed of reading source language. Parallel processing can be found here. In reading translated source language, the homologous words are easily to be activated. Bilingual psychology is similar to the effect of homologous words. The homologous words are automatic, unconscious and non-strategic. So the similarity of words will influence the degree of language activation. The higher the degree of similarity, the higher the degree of source language activation and parallel processing. Compared with English-Spanish translation, English-Chinese is very different. So, in the reading of translated source language, English has a relatively low level of activation, which is the asymmetrical parallel processing due to the differences in interpretation.

Second, in the process of interpreting, language proficiency and parallel processing level is closely related. A full analysis of the asymmetric representation model of bilingual mental lexicon makes it clear that for asymmetric bilingual in lexical level, the connection between mother tongue and second language is of higher degree than the reverse connection. For the connection between concepts and vocabulary, the connection between the concept and the second language be lower than that of concept and the mother tongue. For bilingual, the second vocabulary is more likely to activate than the mother tongue, and of more advantages in the activation of concept-representing mother tongue. When the mother tongue is interpreted proficiently, it will be less processed. On the contrary, it is more processed. Different degree of proficiency will also cause asymmetric processing.

Third, working memory also affects the parallel processing of spoken language transfer. Working memory is an important cognitive psychology, which influences interpreting performance and language understanding. Readers with high working memory has more clear reasoning. With inconsistent reasoning and language input, the reader needs to reason forward and re-read. It can be found that translators with high working memory will not start working on the structure or the words if they are unsure. Those with low working memory will start language transfer. Working memory is very important. The degree of working memory will lead to asymmetric processing ${ }^{[2]}$.

\section{Activation in Processing and Output in Result}

Processing during language transfer is not equivalent to objecting to the consecutive language transfer, not to a certain extent, affecting the integrity of deverbalization. It can be analyzed from two aspects. First, in the online study of psychological linguistics, structure and vocabulary will be activated in the process of understanding source language. It is a kind of unconscious and automatic processing. This processing method has the potential to make a delay in actual understanding, but not affecting the integrity of source language. It is the same as understanding the ambiguous vocabulary in mother tongue. According to the studies of scholars, a high level of bilingual ability can automatically process the ambiguous words in sentences, and can actually find and match the words that are not consistent with the context to integrate the word meaning. It can also be said that in understanding source language, the associative meaning is automatically activated, and the true 
meaning of source language will be affected. Second, In processing the bilingual language, a language is activated, and the other is chosen. Or in understanding the source language, the activation of target language structure and words is not required to be chosen. If there is no choice, there is no need to transfer. So, it can be discovered if the parallel processing of source language can be completely deverbalization, it can be a unconscious and automatic processing ${ }^{[3]}$.

In language transfer in interpreting, automatic processing of language and language fluency has a great relationship. Currently, what the online studies of psychological language have found is automatically processed language, hence fluent bilinguals and professional translators are needed. In automatic processing, early activation of the target language has some effect. But if the target language cannot be automatically activated, the activation of the target language will consume a certain amount of cognitive resources, and will affect the full understanding of the target language, and then appear partial code transfer will appear. When the experts studying the lexical ambiguity are given proof to find out whether the consistences of English context and lexical ambiguity are with different process, they found that, first, low level of tested unambiguous interpretation is controlled, while high level not, which belongs to the automatic process; second, if the tested information has inhibiting ability, the high level of inhibition ability is better than the low level. A large number of experimental data shows that high level testing has the ability to automatically activate the ambiguous vocabulary. It can quickly inhibit the unrelated word meaning, and not affect the understanding of the source language. It can be found that, in the understanding of the source language, for subjects with high level, automatic activation of relevant interpreting is inevitable, but will not affect the overall understanding of the source language. But for those with low level, activation of language is less possible. Based on the above problems, it is not difficult to understand the source language, but difficult to express the target language. If there are other reasons for not activating the target language, a certain amount of cognitive resources will be consumed, the understanding of source language will be affected, so as to a large extent affect the translation quality. A coherent translation fragments and code transfer will not be formed. If the interpreter is fluent in bilingual, he/she can be can change an asymmetric model to a symmetric one for processing, and it can be called a limited parallel processing. For bilingual interpreters, there is no big difference in the difficulty of translating from the mother tongue to the second language, or from the second language to the native language. It is not very difficult to turn the mother tongue into a second language. Based on this, the parallel processing of the source language in understanding source language not only results in deverbalized interpreting, but also the target language can be fluently expressed.

In a certain extent, the training of interpreting skills can improve the output of target language and the understanding of source language. First, In understanding the source language, professional translators can automatically activate some words in target language, but can not make deliberate processing and reasonable choices, and therefore, will not affect the understanding of source language. If the interpreter can not control this phenomenon reasonably, the problem of over-processing of target language in understanding the source language will be caused, and code transfer will be formed. It will affect the expression of target language and understanding the source language. Second, according to the studies on psychological linguistic structure, it can be found the interpreter prefers to listen to the sentence structure in front in understanding. For example, some scholars studied the Spanish bilinguals who are familiar with the picture sentence and re-describe it in English. It can be found that interpreters are more like to use the sentence structure heard in front, which means they are also to be able to activate the structures across languages. In expressing another language, bilingual speakers prefer the sentence structure in front, which is not an unconscious tendency. To a certain extent, the interpreter' s problem in controlling the source language structure belongs to the problem of deverbalization. Through the training of bilingual interpreting, the interpreter can store more expressions suitable for reality, which can resist the effect of sentence activation [4]. Language transfer is not only influenced by language level, but also by the training of interpreting ability. The training of interpretation skills will increase the awareness of bilingual structure, and guarantee that the structure and meaning can be automatically transferred, which facilitates the production of target language and understanding of the source language. Adverbials in 
Chinese are basically in front, but the ones in English are relatively free. In understanding and reading English clauses, they cannot be read for a long time. Therefore, the target language structure cannot be activated to understand source language in C-E interpreting and understanding the difference of two languages, so that the target language structure can be produced. The training of interpreting skills can appropriately increase the working memory ability, so as to avoid excessive local processing and non-coherent inference of target language, so it is helpful for understanding and processing of target language. In actual cases, there may be other reasons making working memory conducive to the expression of interpretation. For example, simultaneous interpreters are dependent on the central actuators, which means target language production and comprehension of the source language can be better controlled ${ }^{[5]}$.

\section{Conclusion}

In a word, in the understanding and analysis of language transfer, the analysis of language conversion, parallel and serial and deverbalization are analyzed, and an asymmetric finite parallel processing method has been proposed, which is normal. Language similarity and parallel processing level are proportional to language fluency, and inversely proportional to working memory. Over parallel processing will affect language transfer and interpreting quality. Improve language fluency can effectively improve the overall quality and promote language transfer.

\section{References}

[1] Xu Han. Cognitive Load in Information Processing in Consecutive Interpreting and Coping Strategies. Journal of Nanchang University (Humanities and Social Science), 2013, 44(6): 152-157.

[2] Gao Lulu, Zhu Yuncui. On Deverbalization in Consecutive Interpreting from the Perspective of Interpretive Theory. Journal of Northeast Normal University (Philosophy and Social Science),2013(6):177-180.

[3] Zhang Jiliang. On Differences between Consecutive Interpreting and Simultaneous Interpreting.Shanghai Journal of Translators for Science and Technology, 2012 (1): 33-36.

[4] Wang Xiaolu, Ji Rongqin. On Relations between Listening Ability Training and Improvement of Consecutive Interpreting Quality -- Based on Empirical Studies of Cognitive Load Model in Consecutive Interpreting. Audio-visual Teaching of Foreign Language, 2015(2):42-46.

[5] Tan Yanzhen, Zhu Yeqiu, Yan Yan. A Contrastive Study of Pause Position in C-E Consecutive Interpreting. Foreign Language and Literature (Journal of Sichuan International Studies University), 2013,29(3):122-127. 